\title{
La Vulnerabilidad del Capital Humano en la Vialidad Lima Metropolitana y el Callao
}

The Vulnerability Of Human Capital In The Viality

In Lima Metropolitana And Callao

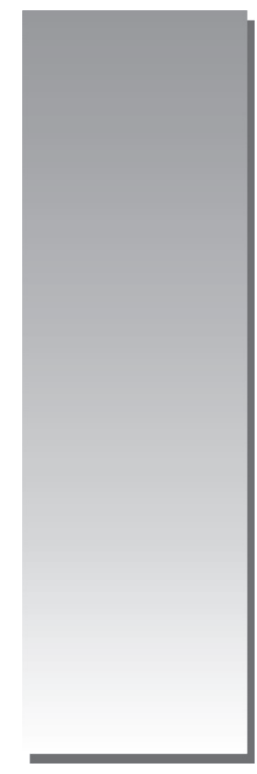

Édgar Vicente Armas edgarvicente02@gmail.com

[RECEPCIÓN: AGOSTO 2016 / CONFORMIDAD: OCTUBRE 2016]

\section{RESUMEN}

En Lima y Callao, el 25\% (4.2 millones) de los 16 millones de viajes diarios se realizan mediante la modalidad de caminata. De este total, el $49.2 \%$ ha sido privado, $35.3 \%$ al colegio y $11.9 \%$ al trabajo; del total privado por el modo caminante, el $52 \%$ ha sido para realizar compras y en menor porcentaje las actividades de entretenimiento y salir a comer (restaurantes). Estas son personas jóvenes (estudiantes), amas de casa y población de menor recurso económico. Se concentran en los lugares donde residen mayoritariamente las personas en pobreza, es decir, en las periferias de la metrópoli: distritos de Comas, Ate Vitarte, San Juan de Lurigancho y otros. Pese a su gran importancia, de este modo, se ha convertido en lo más peligroso; de cada 100 personas muertas por accidentes de tránsito, 78 fueron peatones. Existen otros factores que condicionan a la acción del peatón, tales como: Semaforización peatonal insuficiente, conflicto de flujos vehiculares con flujos peatonales en el momento de giro a la derecha e izquierda, momentos de luz ámbar, entre otros.

Palabras clave: Caminata, tránsito, accidente, vulnerabilidad, peatón.

\section{ABSTRACT}

In the Metropolitan City of Lima and Callao, 25\% (4.2 Million) of the 16 million daily trips are made through the walk mode, of this total, $49.2 \%$ have been private, $35.3 \%$ to the school and $11.9 \%$ to work; Of the total private by the way walker $52 \%$ has been to make purchases and to a lesser percentage the activities of entertainment and to go out to eat (restaurants). The ones that make use are young people (students), housewives and population of smaller economic resource. Concentrate on the places where the majority of people live in poverty, that is to say, in the outskirts of the metropolis, districts of Comas, Ate Vitarte, San Juàn de Lurigancho and others. In spite of its great importance in this way, it has become the most dangerous; Of every 100 people killed by traffic accidents 78 were pedestrians. There are other factors that condition pedestrian action such as: Insufficient pedestrian traffic; Conflict of vehicular flows with pedestrian flows in the moment of rotation to the right and left among others. $\mathrm{N}$ moments of amber light among others.

Key words: Walk, transit, accident, vulnerability, pedestrian.

\footnotetext{
Doctor en Administración, Magíster en Administración con Mención en Gestión Empresarial, Docente asociado de pregrado y posgrado de la Facultad de Ciencias Administrativas de la Universidad Nacional Mayor de San Marcos.
} 


\section{INTRODUCCIÓN}

La presente investigación tiene como finalidad describir que la caminata es uno de los modos de transporte más importante de la ciudad". El 25\% de los 16 millones de viajes diarios se realizan mediante este modo. Es usado principalmente con propósitos de ir al colegio y realizar compras, por lo que pueden estar compuestos por personas jóvenes (estudiantes), amas de casa y población de menor recurso económico. Pese a su gran importancia en la movilidad de la ciudad, este modo de transporte se muestra sumamente peligroso respecto a su vialidad. Según la Secretaría Técnica del Consejo de Transporte de Lima y Callao (STCTLC), los atropellos representan casi el $30 \%$ de la totalidad de los accidentes $(40,390$ accidentes evaluados en el estudio que corresponde al Área metropolitana de Lima y Callao) y, al mismo tiempo, representan el $78 \%$ de las muertes registradas por accidentes. Los objetivos de la investigación son (1) mostrar una realidad vigente poco entendida y conocida de la vulnerabilidad del capital humano en las vías de la ciudad, a fin de sensibilizar la atención de las autoridades nacionales, regionales y municipales sobre este grave problema, y que los mismos asuman su real responsabilidad en el tema. (2) Mostrar la importancia del modo de caminata en el área metropolitana de Lima y Callao y la falta de condiciones de seguridad vial que enfrentan los peatones en sus desplazamientos por las vías de la ciudad.

(3) Mostrar la vulnerabilidad del peatón como una realidad vigente, poco entendida y atendida por las autoridades locales. Evidenciar las características de este frente a la infraestructura vial y la complejidad del tránsito y modos de transporte en la ciudad.

\section{MÉTODOS}

El método empleado es el no experimental, descriptivo de análisis y síntesis; las fuentes de información son básicamente secundarias, de entidades oficiales nacionales responsables.

\section{RESULTADOS \\ CAMINAR: UN IMPORTANTE MODO DE TRANSPORTE}

De un total de 16.53 millones de viajes diarios, el transporte público representa el 51.5\% de los viajes, mientras que los privados y los taxis colectivo en conjunto, el 23\%. Sin embargo, de manera resaltante, el modo de caminata representa el $25 \%$ de los viajes realizados en la metrópoli, lo que equivale decir 4.2 millones de viajes diarios.

Cuadro 1. Distribución de viajes según modo en Lima y Callao al 2010

\begin{tabular}{|c|c|c|}
\hline Modo de transporte & Viajes (En Miles) & $\%$ \\
\hline Caminata & $\mathbf{4 , 2 0 8}$ & $\mathbf{2 5 . 5}$ \\
\hline Privado & 2,092 & 12.7 \\
\hline Vehículos & 1,856 & 11.2 \\
\hline Otros & 236 & 1.4 \\
\hline Taxi-Colectivo & 1,684 & 10.2 \\
\hline Mototaxi & 600 & 3.6 \\
\hline Colectivo & 181 & 1.1 \\
\hline Taxi & 903 & 5.5 \\
\hline Público & 8,525 & 51.6 \\
\hline Combi & 3,791 & 23.0 \\
\hline Microbus & 3,072 & 18.6 \\
\hline Bus & 1,661 & 10.1 \\
\hline Total & 16,508 & 100.0 \\
\hline
\end{tabular}

Fuente: Plan de Transporte Urbano Lima-Callao 2010

Los viajes que se dan por caminata, en la metrópoli Lima-Callao, son motivados principalmente por actividades privadas (ir de compras, salida a restaurantes, entretenimiento, etc.) y para ir al colegio. El primero de los motivos representa el $49 \%$ del total de viajes de caminata; mientras que el segundo, el 35\% de los viajes. 
Cuadro 2. Distribución de los viajes diarios y otros modos según propósito año 2010

\begin{tabular}{|l|c|c|c|c|c|c|}
\hline & \multicolumn{2}{|c|}{$\begin{array}{c}\text { Viajes diarios en todos los } \\
\text { modos }\end{array}$} & \multicolumn{2}{c|}{ Viajes en modo caminata } & \multicolumn{2}{c|}{ Viajes en vehículos } \\
\hline \multicolumn{1}{|c|}{ Propósito de viaje } & En miles & $\%$ & En miles & $\%$ & En miles & $\%$ \\
\hline Al trabajo & $\mathbf{2 , 6 7 7}$ & $\mathbf{3 0 . 5}$ & $\mathbf{2 6 4}$ & $\mathbf{1 1 . 9}$ & $\mathbf{2 , 4 1 3}$ & 36.7 \\
\hline Al colegio & $\mathbf{2 , 3 0 0}$ & $\mathbf{2 6 . 2}$ & $\mathbf{7 8 1}$ & $\mathbf{3 5 . 3}$ & $\mathbf{1 , 5 1 9}$ & $\mathbf{2 3 . 1}$ \\
\hline Negocios & $\mathbf{5 1 1}$ & $\mathbf{5 . 8}$ & $\mathbf{7 8}$ & $\mathbf{3 . 5}$ & $\mathbf{4 3 3}$ & $\mathbf{6 . 6}$ \\
\hline Para negocio & 383 & 4.4 & 35 & 1.6 & 348 & 5.3 \\
\hline Regresar a la oficina & 128 & 1.5 & 42 & 1.9 & 86 & 1.3 \\
\hline Privado & $\mathbf{3 , 2 9 4}$ & $\mathbf{3 7 . 5}$ & 1088 & 49.2 & $\mathbf{2 , 2 0 6}$ & $\mathbf{3 3 . 6}$ \\
\hline Compras & 1,248 & 14.2 & 571 & 25.8 & 677 & 10.3 \\
\hline Restaurante & 151 & 1.7 & 58 & 2.6 & 93 & 1.4 \\
\hline Entretenimiento & 164 & 1.9 & 55 & 2.5 & 109 & 1.7 \\
\hline Recoger o dejar alguien & 311 & 3.5 & 126 & 5.7 & 185 & 2.8 \\
\hline Otros & 1,420 & 16.2 & 278 & 12.6 & 1,142 & 17.4 \\
\hline De retorno a casa & $\mathbf{7 , 7 5 6}$ & & 1,997 & & $\mathbf{5 , 7 5 9}$ & \\
\hline Total & 16,538 & 100.0 & $\mathbf{4 , 2 0 8}$ & 100.0 & 12,330 & \\
\hline
\end{tabular}

Fuente: Plan Maestro de Transporte Urbano para el área metropolitana de Lima y Callao 2005-2015-MTC (ST. CTLC)

De los 1.088 millones de viajes diarios privados y por modo caminata, el $52 \%$ se realiza por compras y, en menor porcentaje, las actividades de entretenimiento y salir a comer (restaurantes).

Como es de esperarse, el modo de caminata es particularmente alto en la población en pobreza, ocupando el $40.5 \%$ del total de los viajes que esta población realiza (900 mil viajes). Este valor es más significativo que el que presenta el resto de la población (se indicaría la población que no se encuentra en pobreza), cuya partición corresponde el $23.2 \%$. Para la población en pobreza, la caminata es el modo de transporte más conveniente dadas sus limitadas posibilidades para acceder a los otros modos (motorizados y no motorizados), ya sea por sus restricciones económicas y/o por la accesibilidad de los servicios de transporte público.

Cuadro 3. Distribución de los viajes de la población en pobreza y resto de la población según modo de transporte 2006

\begin{tabular}{|c|c|c|c|c|c|c|}
\hline & \multicolumn{2}{|c|}{$\begin{array}{c}\text { Viajes diarios en todos los } \\
\text { modos }\end{array}$} & \multicolumn{2}{c|}{ Viajes en modo caminata } & \multicolumn{2}{c|}{ Viajes en vehículos } \\
\hline \multicolumn{1}{|c|}{ Modo de viaje } & En miles & $\%$ & En miles & $\%$ & En miles & $\%$ \\
\hline Caminata & 4,308 & 25.2 & 900 & 40.5 & 3,308 & 23.2 \\
\hline Privado & 2,092 & 12.7 & 52 & 2.3 & 2,040 & 14.3 \\
\hline Carro & 1,856 & 11.2 & 31 & 1.4 & 1,825 & 12.8 \\
\hline Otros & 236 & 1.4 & 21 & 0.9 & 215 & 1.5 \\
\hline Taxi-Colectivo & 1,683 & 10.2 & 182 & 8.2 & 1,501 & 10.5 \\
\hline Mototaxi & 600 & 3.6 & 119 & 5.4 & 481 & 3.4 \\
\hline Colectivo & 181 & 1.1 & 27 & 1.2 & 154 & 1.1 \\
\hline Taxi & 903 & 5.5 & 36 & 1.6 & 867 & 6.1 \\
\hline Público & $\mathbf{8 , 5 2 5}$ & $\mathbf{5 1 . 6}$ & 1089 & 49.0 & 7,436 & $\mathbf{5 2 . 1}$ \\
\hline Combi & 3,791 & 23.0 & 527 & 23.7 & 3,264 & 22.8 \\
\hline Microbús & 3,072 & 18.6 & 352 & 15.8 & 2,720 & 19.0 \\
\hline Bus & 1,661 & 10.1 & 210 & 9.4 & 1,451 & 10.2 \\
\hline Total & 16,508 & 100.0 & 2223 & 100.0 & 14,285 & 100.0 \\
\hline
\end{tabular}

Fuente: Plan Maestro de Transporte Urbano para el área metropolitana de Lima y Callao 2005-2015-MTC (ST. CTLC) 
La población en pobreza, según las estimaciones del Plan Maestro, llegaría aproximadamente al $15 \%$ de la población total de la metrópoli (para el año 2004 el plan estimó para Lima y Callao una población de 8.04 millones de personas), que tiene una tasa de viajes por habitante de 1.8 , mientras que el resto de la población tiene una tasa de 2.1

Ante esto, es de esperar que los viajes por modo caminata se concentren en los lugares donde residen mayoritariamente las personas en pobreza, es decir mayoritariamente en las periferias de la metrópoli. La ilustración que seguido se muestra detalla el ratio de viajes en caminata (respecto al total de viajes) por zonas de tránsito, en ella se puede observar que la zona central tiene ratios de caminata por lo general muy bajos (menores de 0.20), en comparación con los que se observa en las periferias, principalmente en la zona norte de la ciudad.

\section{LOS ACCIDENTES POR ATROPELLOS}

Si bien la caminata es el modo más usado de transporte en la metrópoli, es el más peligroso. El estudio "Análisis de los Accidentes de Tránsito en el Área Metropolitana de Lima y Callao al año 2006" ha arribado a conclusiones dramáticas y preocupantes. Así de cada 100 personas muertas por accidentes de tránsito, 78 fueron peatones. De un total de 40,390 accidentes de tránsito (AC), 11,796 fueron atropellos, es decir, el $29.2 \%$ del total. Cifra importante, pero no tan dramática si la comparamos con la proporción en accidentes que fueron fatales, en los cuales el atropello toma el liderazgo con el 79.6\% de los mismos (de un total de 442 accidentes fatales).

Cuadro 4. Número de accidentes según tipo de fatalidad 2006

\begin{tabular}{|l|c|c|c|c|c|c|}
\hline & \multicolumn{2}{|c|}{ Accidente fatal } & \multicolumn{2}{c|}{ Accidente no fatal } & \multicolumn{2}{c|}{ Total de accidentes } \\
\hline \multicolumn{1}{|c|}{ Tipo de accidente } & Cant. & $\%$ & Cant. & $\%$ & \multicolumn{2}{c|}{ Cant. } \\
\hline Atropello & 352 & 79.6 & 11,444 & 28.6 & 11,796 & 29.2 \\
\hline Otros & 90 & 20.4 & 28,504 & 71.4 & 28,594 & 70.8 \\
\hline Total General & 442 & 100.0 & 39,948 & 100.0 & 40,390 & 100.0 \\
\hline
\end{tabular}

Fuente: Secretaría Técnica del Consejo de Transporte de Lima y Callao 2005-2015

Las cifras indicadas en la tabla anterior refieren a los accidentes de tránsito (propiamente el evento de tránsito), los cuales generaron un total de 95,637 de víctimas, de ellas, a su vez, 450 resultaron ser fatales $(0.5 \%)$. Los ilesos y los heridos en su conjunto representan el 99.5\% de las víctimas.

Cuadro 5. Distribución de las personas según resultados del accidente año 2006

\begin{tabular}{|l|c|c|}
\hline & \multicolumn{2}{c|}{ Total } \\
\hline \multicolumn{1}{|c|}{ Condición } & Número & $\%$ \\
\hline Ileso & 57,320 & 59.9 \\
\hline Herido & 37,867 & 39.6 \\
\hline Muerto & 450 & 0.5 \\
\hline Total & 95,637 & 100.0 \\
\hline
\end{tabular}

Fuente: Secretaría Técnica del Consejo de Transporte de Lima y Callao
Si analizamos el total de las víctimas en función del tipo de accidente, veremos que el $26 \%$ de las personas victimadas (entre ilesos, heridos y muertos) fueron atropelladas por un vehículo. Mientras que el $62 \%$ corresponde a víctimas por efectos de choques, valores que indicarían que aparentemente los choques son más peligrosos que los atropellos. Sin embargo, si evaluamos a las víctimas fatales (personas muertas), veremos que ante un choque la posibilidad de que una persona muera es del $14.4 \%$, mientras que la posibilidad de salir ileso es del $70.1 \%$, condiciones que no se replican lamentablemente para las personas (refiriéndose a peatones) que son atropelladas. La posibilidad de salir ileso en un atropello es de tan solo el $21.2 \%$, mientras que la posibilidad de morir llega al $78.2 \%$. 
Cuadro 6. Distribución de las personas involucradas en los accidentes de tránsito según condiciones y tipo de accidentes año 2006

\begin{tabular}{|l|c|c|c|c|c|c|c|c|}
\hline & \multicolumn{2}{|c|}{ Accidente Fatal } & \multicolumn{2}{c|}{ Accidente no fatal } & \multicolumn{2}{c|}{ Total de accidentes } & \multicolumn{2}{c|}{} \\
\hline \multicolumn{1}{|c|}{ Condición } & Número & $\%$ & Número & $\%$ & Número & $\%$ & Número & $\%$ \\
\hline Ileso & 12,144 & 21.2 & 40,167 & 70.1 & 5,009 & 8.7 & 57,320 & 100.0 \\
\hline Herido & 12,263 & 32.4 & 49,415 & 51.3 & 6,189 & 16.3 & 37,867 & 100.0 \\
\hline Muerto & 352 & 78.2 & 65 & 14.4 & 33 & 7.3 & 450 & 100.0 \\
\hline Total & $\mathbf{2 4 , 7 5 9}$ & $\mathbf{2 6 . 0}$ & $\mathbf{5 9 , 6 4 7}$ & $\mathbf{6 2 . 0}$ & $\mathbf{1 1 , 2 3 1}$ & $\mathbf{1 2 . 0}$ & $\mathbf{9 5 , 6 3 7}$ & 100.0 \\
\hline
\end{tabular}

Fuente: Secretaría Técnica del Consejo de Transporte de Lima y Callao

\section{CAMINAR ES EL MODO DE TRANSPORTE DE MÁS ALTO RIESGO EN LA CIUDAD}

Podemos medir la peligrosidad de cada modo de transporte, para lo cual tenemos la cantidad de muertes por accidentes de tránsito en el modo caminata (que se entenderá por peatones muertos en atropello), que llega a 352 personas y la cantidad de viajes diarios que se realizan es ese modo, que se encuentra en más de cuatro millones de viajes diarios. Por otro lado, han muerto 98 personas en otros modos de viaje, lo que a su vez suman más de 12 millones de viajes diarios. De esto, obtenemos que por cada 100 mil viajes realizados a pie, mueren por accidentes de tránsito ocho personas; mientras que por cada 100 mil viajes en otros modos, muere menos de una persona por accidente de tránsito.

\section{Cuadro 7. Ratio de muertes por cada 100 mil viajes} según tipo de accidente 2006

\begin{tabular}{|l|c|c|c|}
\hline $\begin{array}{c}\text { Tipo de } \\
\text { accidente }\end{array}$ & $\begin{array}{c}\text { Personas } \\
\text { muertas }\end{array}$ & $\begin{array}{c}\text { Cantidad } \\
\text { de viajes } \\
(\mathbf{1 0 0 , 0 0 0 )}\end{array}$ & $\begin{array}{c}\text { Muertes / 100 mil } \\
\text { Viajes }\end{array}$ \\
\hline Atropello & 352 & 42 & 8.4 \\
\hline Choque-otros & 98 & 123 & 0.8 \\
\hline Total & 450 & 165 & 2.7 \\
\hline
\end{tabular}

Fuente: Secretaría Técnica del Consejo de Transporte de Lima y Callao 2005-2015

En pocas palabras, resulta 10 veces más riesgoso movilizarse caminando en la ciudad que ir al interior de un vehículo (como conductor o pasajero), situación que es penosamente resaltante y demuestra la vulnerabilidad del peatón frente al vehículo, por las diferentes avenidas y calles de la urbe.

\section{¿ES EL PEATÓN EL CAUSANTE DE TANTOS ATROPELLOS?}

Según datos estadísticos de la PNP, y cuyo extracto corresponde a los que posee la STCTLC. Estos datos ${ }^{-1}$ sobre la causa generadora del accidente de tránsito responsabiliza al peatón en un 53\% de los casos (atropello), aduciendo su imprudencia. En el $46 \%$ restante de los casos, la responsabilidad recae sobre el conductor de los vehículos (por diferentes aspectos).

Cuadro 8. Causas atribuibles a los atropellos 2006

\begin{tabular}{|c|c|c|}
\hline Causa & $\begin{array}{c}\text { Cantidad de } \\
\text { atropellos }\end{array}$ & $\%$ \\
\hline No específica & $5,573(47 \%)$ & \\
\hline Específica & $6,223(53 \%)$ & 100 \\
\hline Impudencia del peatón & 3,269 & 53 \\
\hline Imprudencia del conductor & 2,320 & 37 \\
\hline Exceso de velocidad & 305 & 5 \\
\hline Ebriedad del conductor & 235 & 4 \\
\hline Otros & 94 & 1 \\
\hline Total & 11,796 & \\
\hline
\end{tabular}

Fuente: Secretaría Técnica del Consejo de Transporte de Lima y Callao 2005-2015

En el área metropolitana de Lima y Callao se registraron 11,796 atropellos (con un total de 12,701 personas atropelladas), de los cuales 6,223 tienen claramente definida la causa del accidente. De los 6,223 accidentes antes referidos, el 53\% acusa la responsabilidad del atropello al peatón, y un $37 \%$ a la imprudencia del conductor.

Los distritos de San Juan de Lurigancho y Cercado de Lima registraron la mayor cantidad de atropellos durante el año 2006, con 1,606 y 1,493 peatones respectivamente, quedando el $2 \%$ muertos y $95 \%$ heridos.

\footnotetext{
***: Se anota que los datos a los que se hace referencia corresponden al $53 \%$ de los datos de accidentes de tránsito de la PNP y que posee la STCTLC. El $47 \%$ restante no indica con claridad la causa del accidente, por lo que no fue tomado para las estadísticas mostradas.
} 
Cuadro 9. Ranking de los 10 distritos con mayor cantidad de atropellos 2005

\begin{tabular}{|c|l|c|c|c|c|c|}
\hline \multicolumn{1}{|c|}{ Distrito } & Atropellos & Peatones & Ileso & Herido & $\begin{array}{c}\text { Peatones } \\
\text { Muertos }\end{array}$ \\
\hline 1 & San Juan de Lurigancho & 1429 & 1606 & 66 & 1517 & 23 \\
\hline 2 & Cercado de Lima & 1374 & 1493 & 57 & 1408 & 28 \\
\hline 3 & Comas & 775 & 831 & 10 & 785 & 36 \\
\hline 4 & La Victoria & 687 & 732 & 12 & 702 & 18 \\
\hline 5 & San Martín de Porres & 644 & 683 & 14 & 646 & 23 \\
\hline 6 & Ate Vitarte & 514 & 546 & 2 & 506 & 38 \\
\hline 7 & Callao & 511 & 532 & 0 & 506 & 17 \\
\hline 8 & Santiago de Surco & 401 & 429 & 10 & 402 & 17 \\
\hline 9 & Independencia & 386 & 416 & 6 & 398 & 12 \\
\hline 10 & San Juan de Miraflores & 376 & 403 & 5 & 389 & 9 \\
\hline
\end{tabular}

Fuente: Secretaría Técnica del Consejo de Transporte de Lima y Callao 2005-2015

Los puntos con mayor cantidad de atropellos se han registrado en las intersecciones de la Av. Próceres, Av. Santiago de Surco, Av. Los Héroes, Av. San Juan, en los distritos de Santiago de Surco y San Juan de Miraflores respectivamente, y sobre ellos se ha evaluado, in situ, las causas probables de atropellos.

Cuadro 10. Ranking de los 10 puntos de ocurrencia de mayor cantidad de atropellos 2006

\begin{tabular}{|c|l|l|c|c|c|}
\hline $\mathbf{N}^{0}$ & \multicolumn{1}{|c|}{ Distrito } & \multicolumn{1}{|c|}{ Ubicación } & Atropellos & Peatones & Muertos \\
\hline 1 & Santigo de Surco & Av. Próceres Plaza Vea & 53 & 56 & 1 \\
\hline 2 & Comas & Av. Panamericana (Norte-Sur) Pro & 34 & 37 & 5 \\
\hline 3 & Independencia & Av. Mendiola (Norte-Sur) Pro Los Olivos & 34 & 34 & 3 \\
\hline 4 & Ate Vitarte & Av. Carretera Central Mercado Ceres & 31 & 31 & 1 \\
\hline 5 & San Juan de Lurigancho & Av. Wiesse Paradero 5 (Este-Oeste) & 31 & 31 & 1 \\
\hline 6 & Comas & Av. Tupac Amaru Auxiliar Inka farma Av. Belaunde & 26 & 28 & 2 \\
\hline 7 & Ate Vitarte & Av. Carretera Central km 6 1/2 & 23 & 27 & 3 \\
\hline 8 & San Juan de Lurigancho & Av. Próceres de la Independencia Cuadra 16 & 23 & 26 & 2 \\
\hline 9 & Santiago de Surco & Av. Tomás Marsano a 100mts Puente Atocongo & 22 & 25 & 3 \\
\hline 10 & Cercado de Lima & Av. Grau Av. Paseo Colón & 24 & 24 & 1 \\
\hline
\end{tabular}

Fuente: Secretaría Técnica del Consejo de Transporte de Lima y Callao

Previo a mostrar la evaluación realizada en campo, en los dos puntos arriba indicados, en conjunto registraron 101 atropellos (con un total de 105 personas atropelladas), de los cuales 43 tienen claramente definida la causa del accidente. De los 43 accidentes antes referidos, el $77 \%$ acusa la responsabilidad del atropello al peatón.

\section{EL TRANSPORTE PÚBLICO ES EL QUE GENERA MAYOR CANTIDAD DE MUERTES POR ATROPELLO}

Según la STCTLC, el 36\% de los peatones muertos por atropello fueron ocasionados por vehículos de transporte público (ómnibus, microbús y camioneta rural), proporción sorprendentemente mayor al de los autos, los cuales ocasionaron el $34 \%$ de la mortandad de los peatones. En Lima hay 
un poco más de 29 mil vehículos de transporte público versus el más de un millón de vehículos privados existentes (Plan Maestro de Transporte Urbano para Lima y Callao). Según esto, podemos señalar que el 3\% de vehículos de la ciudad (que corresponden al transporte público) producen el $36 \%$ de muertes por atropello. Las autoridades municipales y provinciales deberían tomar acciones contundentes para reducir estas dramáticas cifras, por estar dentro de su plena competencia. Se debe anotar que el análisis hecho en este punto corresponde al $85 \%$ de la data de peatones muertos. El 15\% faltante no registró datos de tipo de vehículo que atropellaba.

\section{Cuadro 11. Distribución de muertes por atropello} según vehículo responsable

\begin{tabular}{|l|c|c|}
\hline \multicolumn{1}{|c|}{ Vehículo } & $\begin{array}{c}\text { Número de } \\
\text { muertos }\end{array}$ & $\%$ \\
\hline No Específica & $52(14.7 \%)$ & \\
\hline
\end{tabular}

\begin{tabular}{|l|c|c|}
\hline \multicolumn{1}{|c|}{ Específica } & $\mathbf{3 0 0}(\mathbf{8 5 . 3 \% )}$ & $\mathbf{1 0 0}$ \\
\hline Microbús & 6 & 2.0 \\
\hline Ómnibus & 64 & 21.3 \\
\hline Camioneta Rural & 37 & 12.3 \\
\hline Auto & 104 & 34.7 \\
\hline Furgoneta & 1 & 0.3 \\
\hline Camión & 32 & 10.7 \\
\hline Moto & 2 & 0.7 \\
\hline Camioneta & 28 & 9.3 \\
\hline Trailer & 4 & 1.3 \\
\hline Mototaxi & 15 & 5.0 \\
\hline Otro & 7 & 2.3 \\
\hline Total & 352 & \\
\hline
\end{tabular}

Fuente: Secretaría técnica del consejo de trasporte de Lima y Callao

Cuadro 12. Muertes por atropello y número de vehículos según tipo 2006

\begin{tabular}{|l|c|c|c|c|}
\hline Tipo de vehículo & $\begin{array}{c}\text { Muertes por } \\
\text { atropello }\end{array}$ & $\%$ & $\begin{array}{c}\text { Número de } \\
\text { vehículo } \\
\text { (Miles) }\end{array}$ & $\%$ \\
\hline $\begin{array}{l}\text { Transporte Público } \\
\text { (Bus, Microbús, } \\
\text { Camioneta Rural) }\end{array}$ & 107 & 36 & 29 & 3 \\
\hline Autos & 104 & 34 & 386 & 35 \\
\hline Otros & 89 & 30 & 677 & 62 \\
\hline Total & $\mathbf{3 0 0}$ & 100 & 1,092 & 100 \\
\hline
\end{tabular}

Fuente: Secretaría Técnica del Consejo de Transporte de Lima y Callao

\section{LAS PERSONAS CON EDAD QUE CONFORMAN LA POBLACIÓN ECONÓMICAMENTE ACTIVA PRESENTAN MAYOR RIESGO DE MUERTE}

Si profundizamos el análisis en el perfil de las personas que son atropelladas, encontraremos que, para el área metropolitana, las personas que mayormente son atropelladas se encuentran entre los 19 y 40 años de edad (36\% del total); sin embargo, la mortalidad afecta casi de la misma forma a las personas que están entre los 19-40 años, 41-60 años y más de 61 años $(30 \%, 28 \%$ y $32 \%$, respectivamente), debiendo anotar que entre 19-60 años, conformantes de la PEA, es el 58\% y mayor de 60 años el $28 \%$.

Cuadro 13. Distribución de los peatones atropellados según rango de edad 2006

\begin{tabular}{|c|c|c|c|c|c|c|c|}
\hline $\begin{array}{c}\text { Rango de } \\
\text { edad }\end{array}$ & llesos & Heri & & Mue & & \multicolumn{2}{|c|}{$\begin{array}{c}\text { Total personas } \\
\text { atropelladas }\end{array}$} \\
\hline No Específica & 25 & 400 & & 31 & - & $497(4 \%)$ & \\
\hline
\end{tabular}

\begin{tabular}{|l|c|c|c|c|c|c|c|c|}
\hline Específica & 347 & $100 \%$ & 11,537 & $100 \%$ & 320 & $100 \%$ & $\begin{array}{c}\mathbf{1 2 , 2 0 4} \\
(\mathbf{9 6 \% )}\end{array}$ & $\mathbf{1 0 0 \%}$ \\
\hline $0-5$ años & 2 & 1 & 1,012 & 9 & 7 & 2 & 1,021 & 8 \\
\hline 6-12 años & 3 & 1 & 1,642 & 14 & 7 & 2 & 1,652 & 14 \\
\hline 13-18 años & 9 & 3 & 1,219 & 11 & 20 & 6 & 1,248 & 10 \\
\hline 19-40 años & 231 & 67 & 4,019 & 35 & 96 & 30 & 4,346 & 36 \\
\hline 41-60 años & 89 & 26 & 2,148 & 19 & 89 & 28 & 2,326 & 19 \\
\hline 61 a más & 13 & 4 & 1,497 & 13 & 101 & 32 & 1,611 & 13 \\
\hline Total & 372 & - & 11,977 & - & 352 & - & 12,701 & \\
\hline
\end{tabular}

Fuente: Secretaría Técnica del Consejo de Transporte de Lima y Callao

El hecho de que la mayor cantidad de personas atropelladas se encuentre entre los 19 y 40 años de edad se explica por la mayor cantidad de actividades económicas y sociales que se realiza en ese grupo de edad, lo que implica una mayor exposición de este grupo respecto al total, debiendo anotar que es aquí donde se encuentra mayormente la población económicamente activa. Al respecto, se puede señalar que los atropellos están afectando nuestra masa crítica productiva y, por tanto, nuestra economía global.

\section{SE OBSERVA UNA MAYOR FATALIDAD EN HORAS DE LA MADRUGADA}

La gravedad de consecuencias de los atropellos se incrementa de acuerdo a la hora del día, según se puede observar en los cuadros y gráficas seguidas a continuación. La mayor cantidad de 
peatones atropellados, el $72.5 \%$ del total, son registrados en horas del día (entre las 7:00 y 19:00 horas), mientras que en horas de la noche (entre las 19:00 y 7:00 horas) el porcentaje es menor de $27.4 \%$, es decir, que es más probable (probabilidad de 0.67 ) ser atropellado en las horas de la mañana que en horas de la noche; sin embargo, cuando evaluamos el nivel de gravedad del atropello, estas condiciones varían.

Cuadro 14. Distribución del total de peatones atropellados según turno del día 2006

\begin{tabular}{|l|c|c|}
\hline \multicolumn{1}{|c|}{ Turno del día } & $\begin{array}{c}\text { Total de peatones } \\
\text { atropellados }\end{array}$ & $\%$ \\
\hline No Específica & 2,187 & \\
\hline
\end{tabular}

\begin{tabular}{|l|c|c|}
\hline \multicolumn{1}{|c|}{ Específica } & 10,514 & $100 \%$ \\
\hline Específica & 7,026 & $67 \%$ \\
\hline Mañana (7:00-19:00) horas & 3,488 & $33 \%$ \\
\hline Total & 12,701 & \\
\hline
\end{tabular}

Fuente: Secretaría Técnica del Consejo de Transporte de Lima y Callao

De los datos tenemos que la probabilidad de que una persona resulte muerta en un atropello es el doble en horas de la noche que en la mañana, siendo la probabilidad de la mañana del $2 \%$ y en la noche de $4 \%$.

Cuadro 15. Distribución de peatones atropellados según condición y turno del día 2006

\begin{tabular}{|l|l|l|l|l|}
\hline \multicolumn{1}{|c|}{ Turno } & Ilesos & Heridos & Muertos & Total \\
\hline Mañana (7:00-19:00) Horas & 227 & 6,651 & 148 & 7,026 \\
\hline$\%$ & & & & \\
\hline Noche (19:00-7:00) Horas & & & & \\
\hline$\%$ & & & & \\
\hline Total & 327 & 9,892 & 295 & \\
\hline
\end{tabular}

Fuente: Secretaría técnica del consejo de trasporte de Lima y Callao

Si realizamos una revisión de las probabilidades horarias de morir en un atropello (estimado poblacional que resulta de la división de muertos y total de peatones afectados) en el período de 7:00 a 19:00, veremos que existe una concentración en horas de la madrugada, específicamente entre las 00:00 horas y 06:00 horas (ver cuadro 16). Esto puede ser explicado por las velocidades que pueden desarrollar los vehículos en horas de la madrugada, cuando las vías se encuentran prácticamente libres. Si bien la cantidad de atropellos en esas horas es menor, la probabilidad de muerte es significativamente más alta.

\section{DISCUSIÓN}

La caminata es el modo más usado de transporte en la metrópoli, también es el más peligroso. Los resultados del estudio "Análisis de los Accidentes de Tránsito en el Área Metropolitana de Lima y Callao al año 2006" señalan que de cada 100 personas muertas por accidentes de tránsito, 78 fueron peatones.

Del total de víctimas según tipo de accidente, el $26 \%$ de las personas victimadas (entre ilesos, heridos y muertos) fueron atropelladas por un vehículo. Mientras que el $62 \%$ corresponde a víctimas por efectos de choques, lo que indicaría que los choques son más peligrosos que los atropellos. Sin embargo, si consideramos las víctimas fatales (personas muertas), veremos que ante un choque la posibilidad de que una persona muera es del $14.4 \%$, mientras que la posibilidad de salir ileso es del $70.1 \%$, condiciones que no se replican lamentablemente para las personas (refiriéndose a peatones) que son atropelladas. Por lo que, si una persona sufre un accidente tipo choque, tiene una posibilidad del $70 \%$ de salir ileso; mientras que si es atropellada, la posibilidad de que muera es del $78 \%$.

Según STCTLC, la causa generadora del accidente de tránsito responsabiliza al peatón en un 53\% de los casos (atropello), aduciendo imprudencia del mismo, y en el $46 \%$ restante la responsabilidad recae sobre el conductor de los vehículos (por diferentes aspectos); sin embargo, estas cifras también se condicionan con la presencia de otros factores, entre ellos, los relacionados con la infraestructura vial existente. Estos factores son:

- Semaforización peatonal insuficiente, y los pocos semáforos existentes no tienen tiempos de verde destinados para los peatones.

- Conflicto de flujos vehiculares con flujos peatonales en el momento de giro a la derecha e izquierda; no existen tiempos destinados para el cruce de peatones.

- Reducido espacio en las aceras para el volumen peatonal existente.

- Uso de la vía vehicular ante la falta de paraderos.

- Inexistencias y/o mala ubicación de puentes peatonales para los cruces de las vías.

- Inexistencia o escaso mantenimiento de la señalización preventiva o regulatoria. 
- No existe preferencia al peatón en las vías.

- Los vehículos no respetan los cruceros peatonales; aceleración de vehículos en momentos de luz ámbar, entre otros.

Según edades, el $58 \%$ de personas atropelladas se encuentra entre los 19 y 60 años de edad, lo cual afecta a las actividades económicas y sociales que se realiza en ese grupo de edad. Esto implica una mayor exposición de este grupo respecto al total, debiendo anotar que es aquí donde se encuentra mayormente la población económicamente activa. Al respecto, se puede señalar que los atropellos están afectando nuestra masa crítica productiva y, por tanto, nuestra economía global. Lo cual demuestra la vulnerabilidad del capital humano de la economía del país.

El distrito con mayor vulnerabilidad del capital humano es el distrito de San Juan de Lurigancho, dado que guarda relación directa con el número de habitantes en dicho distrito, el asentamiento de mayor número de pueblos jóvenes y la falta de infraestructura como puentes peatonales, señalizaciones y paraderos.

\section{CONCLUSIONES}

1. La caminata es uno de los modos de transporte más usados en la ciudad y es utilizado principalmente por las personas de menores recursos económicos. Pese a su gran importancia, las autoridades municipales no han logrado proveer de mayor seguridad vial a este grupo de personas, siendo estos constantemente víctimas de las deficiencias de la infraestructura y diseño vial, ocupando un lastimoso $30 \%$ de los accidentes de la ciudad.

2. $\quad$ No se puede esperar y/o exigir adecuados comportamientos de seguridad vial a los usuarios de la vía si no se provee facilidades de infraestructura y equipamiento vial sobre cuyos usos se les pueda sancionar. Queda para reflexionar si es que la autoridad local considera el tema de la seguridad vial como un punto ajeno a su agenda de responsabilidades.
3. En el área metropolitana de Lima y Callao, el peatón es el actor de la vía de menor categoría y, por ende, de mayor desprotección, estando por debajo de prioridad sobre cualquier modo motorizado. Se debe priorizar la protección al peatón sobre los vehículos.

4. La vulnerabilidad del peatón frente a los accidentes de tránsito ha quedado en evidencia, y corresponde a las autoridades locales tomar las acciones correctivas del caso para evitar que se siga afectando las vidas humanas de los ciudadanos, y de manera directa seguir afectando la economía local y nacional.

5. Si bien la imprudencia del peatón es una de las mayores causas de accidentes en las vías de la ciudad, existen también otros factores esenciales que limitan la vialidad del peatón, como la escasa infraestructura y equipamiento vial existente, la competencia de los peatones con los vehículos y con el comercio ambulatorio.

6. Las autoridades locales deben dotar una infraestructura y equipamiento vial operativa, entre las que se señala: paraderos adecuados, semáforos peatonales, señalización preventiva, puentes peatonales, ampliación de aceras, mantenimiento de las vías, paraderos, entre otros.

7. Es necesario que las autoridades locales, educativas y medios de comunicación en general promuevan campañas de educación vial en forma permanente, entre las cuales se considere campañas de sensibilización para la población en general, sobre todo en los lugares de mayor concentración de accidentes de los peatones (puntos negros).

\section{REFERENCIAS BIBLIOGRÁFICAS}

Defensoría del Pueblo (2008). El Transporte Urbano en Lima Metropolitana: Un Desafío en Defensa de la Vida Informe Defensorial $\mathrm{N}^{\mathrm{O}} 137$.

Ministerio de Transportes y Comunicaciones (2005). Secretaría Técnica del Consejo 
del Transporte Urbano Lima y Callao Plan Maestro de Transporte Urbano para Lima y Callao (2005 - 2025).

Ministerio de Transportes y Comunicaciones (2008). Secretaría Técnica del Consejo de Transporte de Lima y Callao. Análisis de accidentes de tránsito en el área central de Lima y Callao - Información base del año 2006.
Ministerio de Transportes y Comunicaciones (MTC) (2009). Consejo de Transporte de Lima y Callao. La vulnerabilidad de los peatones en la vialidad del área Metropolitana de Lima y Callao.

Ministerio de Transportes y Comunicaciones (MTC) (2010). Secretaría Técnica del Consejo de Transporte Urbano Lima Callao. El Transporte Urbano Metropolitano de Lima y Callao en Números. 